Supporting Information

for

\title{
The ring opening reaction of chromenes: a photochemical mode-dependent transformation
}

Annapaola Migani ${ }^{\dagger}$, Pier Luigi Gentili ${ }^{\S}$, Fabrizia Negri ${ }^{\Uparrow}$, Massimo Olivucci ${ }^{\dagger, \AA}$, Aldo Romani ${ }^{\S}$, Gianna Favaro ${ }^{\S}$, Ralph S. Becker ${ }^{\#}$

${ }^{\dagger}$ Dipartimento di Chimica, Università di Siena, 53100 Siena, Italy,

${ }^{\S}$ Dipartimento di Chimica, Università di Perugia, Via Elce di Sotto 8, 06123 Perugia, Italy, "Dipartimento di Chimica, Università di Bologna, Via Selmi 2, 40126 Bologna, Italy,

${ }^{\ddagger}$ Centro per lo Studio dei Sistemi Complessi, Università di Siena, via Pendola 37, Siena, Italy,

${ }^{\#}$ Departamento de Engenharia Quimica, Instituto Superior Tecnico, 1049-100 Lisbon, Portugal.

\section{Contents:}

12 pages including: 1. Computational details for Franck-Condon activity computations (comprising Table S1), 2. Reaction coordinate analysis of $\mathrm{C}_{9}-\mathrm{O}_{1}-\mathrm{C}_{2}-\mathrm{C}_{3}$ and $\mathrm{H}-\mathrm{C}_{2}-\mathrm{C}_{3}-\mathrm{H}\left(\mathrm{Et}-\mathrm{C}_{2}-\mathrm{C}_{3}-\mathrm{H}\right)$ torsions (comprising Figure $\mathrm{S} 1$ ), and 14 tables containing the Cartesian coordinates for the CASSCF/6-31G* optimized geometries discussed in the text. 
1. Computational details for Franck-Condon activity computations. We have assumed that the Franck-Condon (FC) mechanism is the main source of intensity. ${ }^{1}$ The simulation of the FC activity is performed by associating a harmonic oscillator to each totally symmetric mode and obtaining the displacement $B_{i}$ for each totally symmetric mode defined as

$$
B_{i}=0.172 \sqrt{v_{i}}\left(\mathbf{x}_{\mathbf{1}}-\mathbf{x}_{0}\right) \sqrt{\mathbf{M}} \mathbf{L}_{\mathbf{i}}
$$

where $v_{i}\left(\mathrm{~cm}^{-1}\right)$ and $\mathbf{L}_{\mathbf{i}}$ are the $i$ th vibrational frequency and normal mode in the state under study ( $S_{1}$ in our case), $\mathbf{M}$ is the matrix of atomic masses (in amu), and $\mathbf{x}_{\mathbf{1}}$, and $\mathbf{x}_{\mathbf{0}}$ are the Cartesian coordinate vectors (in $\AA$ ) defining the equilibrium structure of the two electronic states involved in the transition. (Note that in the text for simplicity the displacement parameter is referred to as $\mathrm{B}_{\mathrm{S} 0, \mathrm{~S} 1}$ ). The $\mathrm{FC}$ activity of each totally symmetric mode is proportional to the $\gamma_{i}$ parameter that is given by

$\gamma_{i}=0.5 B_{i}^{2}$

A summary of the $\mathrm{S}_{1}$ force field and the corresponding $\gamma_{i}$ parameters for $2 \mathrm{H}$ chromene (C), 2,2-diethyl-2H-chromene (DEC), and an ideal chromene (MDEC) when the two ethyl groups are not included explicitly in the calculation but are approximated by 29 amu point masses is provided in Table S1.

\footnotetext{
${ }^{1}$ Negri, F.; Zgierski, M. Z. J. Phys. Chem. 1993, 99, 4318-4326.
} 
Supporting Information for Migani et al., The Ring Opening of Chromenes... 
Table S1. Vibrational frequencies $\left(\right.$ in $\mathrm{cm}^{-1}$ ) of the normal modes in the $\mathrm{S}_{1}$

state and $\gamma$ parameters for the $\mathrm{S}_{0} \rightarrow \mathrm{S}_{1}$ transition of $\mathrm{C}$ and DEC, and

MDEC.

\begin{tabular}{|c|c|c|c|c|c|c|c|c|c|c|c|c|c|}
\hline \multicolumn{4}{|c|}{$\mathrm{C}$} & \multicolumn{4}{|c|}{ MDEC } & \multicolumn{6}{|c|}{ DEC } \\
\hline$v$ & $\gamma$ & $v$ & $\gamma$ & $v$ & $\gamma$ & $v$ & $\gamma$ & $v$ & $\gamma$ & $v$ & $\gamma$ & $v$ & $\gamma$ \\
\hline 137 & 0.01 & 1333 & 0.06 & 64 & 1.00 & 1227 & 0.04 & 51 & 0.37 & 845 & 0.06 & 1567 & 0.00 \\
\hline 145 & 0.80 & 1350 & 0.02 & 118 & 0.18 & 1252 & 0.02 & 93 & 0.04 & 852 & 0.03 & 1570 & 0.00 \\
\hline 217 & 0.00 & 1374 & 0.12 & 192 & 0.09 & 1258 & 0.03 & 101 & 0.03 & 860 & 0.00 & 1615 & 0.00 \\
\hline 318 & 0.06 & 1478 & 0.04 & 253 & 0.04 & 1304 & 0.41 & 133 & 0.00 & 923 & 0.04 & 1632 & \\
\hline 368 & 0.07 & 1527 & 0.00 & 282 & 0.01 & 1321 & 0.07 & 189 & 0.08 & 959 & 0.01 & 1637 & \\
\hline 384 & 0.00 & 1550 & 0.01 & 305 & 0.04 & 1354 & 0.00 & 202 & 0.01 & 1006 & 0.22 & 1643 & 0.00 \\
\hline 451 & 0.00 & 1578 & 0.06 & 342 & 0.04 & 1399 & 0.09 & 222 & 0.04 & 1020 & 0.00 & 1650 & \\
\hline 506 & 0.06 & 1625 & 0.02 & 384 & 0.06 & 1521 & 0.05 & 246 & & 1031 & 0.02 & 1653 & \\
\hline 512 & 0.04 & 1653 & 0.00 & 412 & 0.01 & 1536 & 0.10 & 285 & 0.00 & 1052 & 0.01 & 1656 & \\
\hline 552 & 0.03 & 1664 & & 440 & 0.05 & 1558 & 0.00 & 301 & 0.05 & 1104 & 0.00 & 1667 & 0.00 \\
\hline 583 & 0.00 & 1708 & 0.01 & 476 & 0.01 & 1615 & 0.00 & 315 & 0.01 & 1134 & 0.00 & 1708 & 0.02 \\
\hline 615 & & 1818 & 0.00 & 498 & 0.05 & 1665 & 0.00 & 355 & 0.00 & 1139 & 0.00 & 1817 & \\
\hline 631 & 0.00 & 3213 & 0.00 & 517 & 0.01 & 1707 & 0.02 & 391 & 0.00 & 1186 & 0.00 & 3207 & \\
\hline 678 & 0.00 & 3295 & 0.00 & 576 & 0.03 & 1818 & 0.00 & 419 & 0.01 & 1225 & 0.00 & 3209 & \\
\hline 716 & 0.00 & 3355 & 0.00 & 591 & 0.01 & 3355 & 0.00 & 450 & 0.06 & 1239 & 0.01 & 3213 & \\
\hline 733 & 0.45 & 3371 & 0.00 & 615 & 0.00 & 3371 & 0.00 & 463 & 0.01 & 1255 & 0.07 & 3218 & \\
\hline 806 & 0.03 & 3383 & 0.00 & 632 & 0.00 & 3383 & & 506 & 0.07 & 1299 & 0.32 & 3246 & \\
\hline 807 & 0.05 & 3386 & 0.00 & 655 & 0.01 & 3386 & 0.00 & 520 & 0.02 & 1301 & 0.00 & 3253 & \\
\hline 891 & 0.00 & 3396 & 0.00 & 692 & 0.00 & 3396 & 0.00 & 561 & 0.01 & 1326 & 0.14 & 3271 & \\
\hline 956 & 0.02 & 3410 & 0.00 & 716 & 0.00 & 3410 & 0.00 & 593 & 0.01 & 1350 & 0.02 & 3272 & \\
\hline 983 & 0.08 & & & 740 & 0.42 & & & 614 & & 1399 & 0.07 & 3283 & \\
\hline 1019 & 0.18 & & & 806 & & & & 626 & 0.00 & 1422 & 0.00 & 3299 & \\
\hline 1072 & & & & 862 & 0.06 & & & 636 & 0.00 & 1460 & 0.01 & 3349 & 0.00 \\
\hline 1084 & 0.00 & & & 931 & 0.04 & & & 694 & 0.03 & 1504 & 0.01 & 3371 & 0.00 \\
\hline 1136 & 0.00 & & & 957 & 0.01 & & & 714 & 0.00 & 1519 & 0.00 & 3380 & \\
\hline 1242 & 0.04 & & & 1011 & 0.24 & & & 720 & 0.03 & 1527 & 0.00 & 3384 & \\
\hline 1258 & 0.04 & & & 1094 & & & & 745 & 0.31 & 1545 & 0.12 & 3394 & 0.00 \\
\hline 1298 & 0.43 & & & 1138 & 0.01 & & & 806 & & 1558 & 0.00 & 3410 & 0.00 \\
\hline
\end{tabular}




\section{Reaction coordinate analysis of $\mathrm{C}_{9}-\mathrm{O}_{1}-\mathrm{C}_{2}-\mathrm{C}_{3}$ and $\mathrm{H}-\mathrm{C}_{2}-\mathrm{C}_{3}-\mathrm{H}\left(\mathrm{Et}-\mathrm{C}_{2}-\mathrm{C}_{3}-\mathrm{H}\right)$}

torsions. Inspection of Figure $\mathrm{S} 1$ shows in more detail the behaviour of $\mathrm{C}_{9}-\mathrm{O}_{1}-\mathrm{C}_{2}-\mathrm{C}_{3}$ torsion along the entire reaction coordinate. In the same figure we also show the behavior of the $\mathrm{H}-\mathrm{C}_{2}-\mathrm{C}_{3}-\mathrm{H}\left(\mathrm{Et}-\mathrm{C}_{2}-\mathrm{C}_{3}-\mathrm{H}\right)$ geometrical parameter.

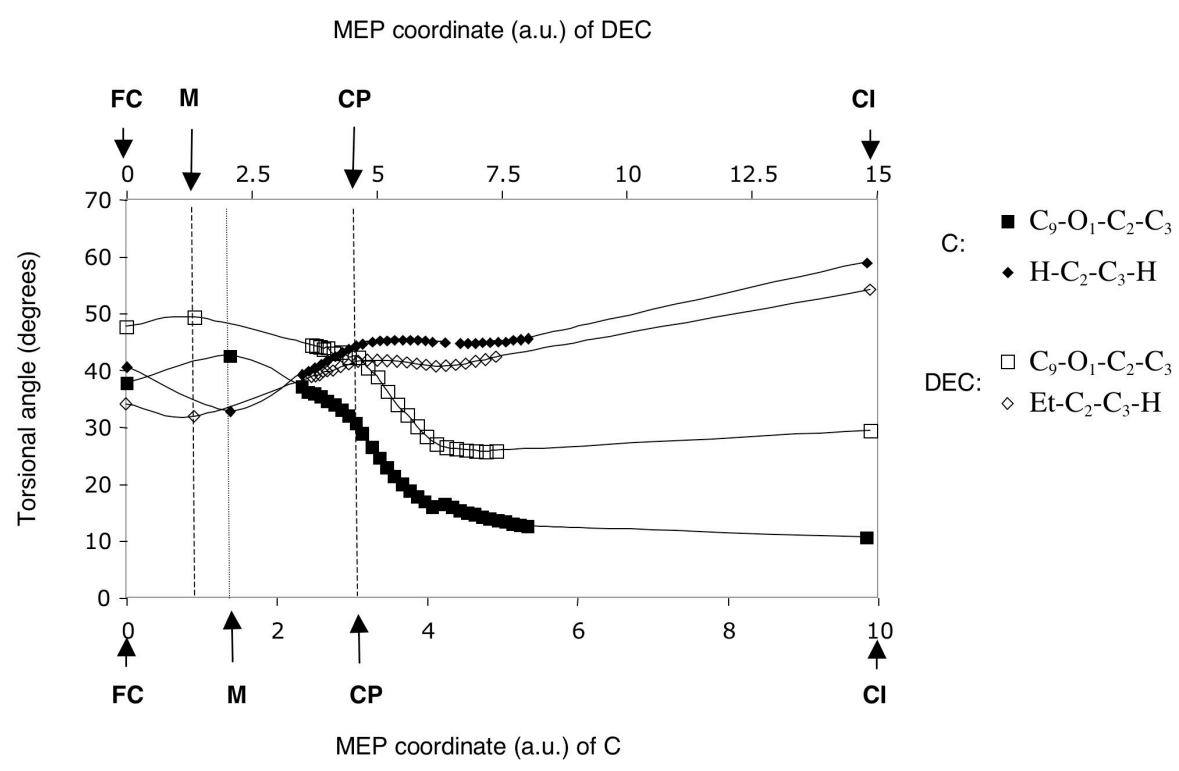

Figure S1

Figure S1. Change in the $\mathrm{C}_{9}-\mathrm{O}_{1}-\mathrm{C}_{2}-\mathrm{C}_{3}$ and $\mathrm{H}-\mathrm{C}_{2}-\mathrm{C}_{3}-\mathrm{H} / \mathrm{Et}^{-} \mathrm{C}_{2}-\mathrm{C}_{3}-\mathrm{H}$ dihedral angles. 
Supporting Information for Migani et al., The Ring Opening of Chromenes...

Tables of Cartesian Coordinates (XYZ) of the optimized structures discussed in the text. Values are given in $\AA$.

\section{H-chromene:}

FC

$\begin{array}{rrr}-0.0429483302 & 0.0265927014 & -0.0095985380 \\ -0.0165285965 & -0.0714397470 & 1.4107162165 \\ 1.2398799754 & -0.1265185025 & 2.0883172544 \\ 2.5003684797 & -0.0582062584 & 1.3903130949 \\ 2.4402549327 & 0.0414941992 & -0.0483935221 \\ 1.1785756133 & 0.0801545573 & -0.7252712324 \\ -0.9796885083 & 0.0562337116 & -0.5318052694 \\ -0.9157136305 & -0.1090510500 & 1.9936722594 \\ 3.3523338628 & 0.1064155259 & -0.6110213536 \\ 1.1654154996 & 0.1563789336 & -1.7966441301 \\ 1.1887318297 & -0.1183343733 & 3.4264696066 \\ 3.6957002380 & -0.0269150995 & 2.1525090773 \\ 3.6571957314 & -0.1988704036 & 3.5061245709 \\ 4.6318609033 & 0.1470091900 & 1.6511899790 \\ 2.3650105912 & -0.5372184953 & 4.1786989351 \\ 2.2640658267 & -0.0465428931 & 5.1363007953 \\ 2.2690120595 & -1.6076111139 & 4.3362672647 \\ 4.5459375224 & -0.1786428824 & 4.1091139916\end{array}$

\section{CP}


Supporting Information for Migani et al., The Ring Opening of Chromenes...

$\begin{array}{lrrr}\mathrm{C} & 1.185775 & .050566 & -.701339 \\ \mathrm{H} & -.968816 & .175767 & -.500546 \\ \mathrm{H} & -.903580 & -.020489 & 2.024556 \\ \mathrm{H} & 3.355755 & -.061635 & -.599187 \\ \mathrm{H} & 1.175054 & .123776 & -1.773151 \\ \mathrm{O} & 1.223543 & -.148913 & 3.448881 \\ \mathrm{C} & 3.714837 & -.049545 & 2.158010 \\ \mathrm{C} & 3.681901 & -.211833 & 3.513136 \\ \mathrm{H} & 4.632468 & .190000 & 1.648688 \\ \mathrm{C} & 2.404993 & -.697004 & 4.119970 \\ \mathrm{H} & 2.290514 & -.407276 & 5.153609 \\ \mathrm{H} & 2.324218 & -1.777803 & 4.054058 \\ \mathrm{H} & 4.550370 & -.096750 & 4.135199\end{array}$

\section{TS}

$\begin{array}{rrr}-.032559 & .090831 & .014910 \\ -.006454 & -.027420 & 1.422300 \\ 1.232403 & -.124429 & 2.114623 \\ 2.516975 & -.151843 & 1.412358 \\ 2.432435 & -.077269 & -.006975 \\ 1.186265 & .050598 & -.685657 \\ -.965672 & .184282 & -.506244 \\ -.905935 & -.032926 & 2.007615 \\ 3.341747 & -.063257 & -.579172 \\ 1.187228 & . .122400 & -1.757870 \\ 1.169192 & -.124782 & 3.410212 \\ 3.710885 & -.070084 & 2.152331 \\ 3.699268 & -.210759 & 3.529847 \\ 4.619245 & .183597 & 1.632526 \\ 2.489038 & -.729533 & 4.133591 \\ 2.328527 & -.486689 & 5.170220 \\ 2.276801 & -1.772205 & 3.945241 \\ 4.498577 & .146325 & 4.151208\end{array}$

CI

$\begin{array}{rrr}.004446 & .052717 & -.008661 \\ -.030754 & .028378 & 1.381568 \\ 1.160489 & -.054962 & 2.128548 \\ 2.424117 & -.108273 & 1.454817 \\ 2.417314 & -.060604 & .051484 \\ 1.232833 & .013392 & -.680105 \\ -.912029 & .090674 & -.567651 \\ -.959763 & .044603 & 1.918157 \\ 3.361211 & -.079809 & -.463992 \\ 1.258707 & .033592 & -1.754182 \\ 1.063038 & -.009150 & 3.441281 \\ 3.679227 & -.074939 & 2.193776 \\ 3.817365 & -.541704 & 3.481291 \\ 4.509362 & .423832 & 1.726487 \\ 2.826518 & -1.325934 & 4.123092 \\ 2.860885 & -1.475538 & 5.186585 \\ 2.209684 & -2.003063 & 3.573014 \\ 4.690069 & -.244675 & 4.039923\end{array}$


Supporting Information for Migani et al., The Ring Opening of Chromenes...

2,2-diethyl-2H-chromene:

\section{exo-exo $S_{0}$ minimum $=F C$}

.039031

.023197

1.224791

2.446889

2.445963

1.250826

$-.889141$

$-.899800$

3. 384512

1. 262245

1.159962

3.662956

3.608127

4.595460

2. 318541

4.502559

2. 092263

3.278804

1.263512

1.751463

2.940727

3. 730987

4.054483

2. 168720

3.207075

2. 184029

1.176098

3.061430

4.222520

3. 120189
.002051

$-.050431$

$-.016771$

.079686

.126959

.086171

$-.026150$

-.110952

.201600

.124170

$-.007904$

.150635

$-.056587$

.380725

$-.438390$

$-.022238$

.325284

.478869

$-.148490$

1. 316244

.959353

-. 464294

1.107980

$-1.967624$

$-2.719327$

$-2.364518$

$-2.164042$

$-3.788183$

$-2.495548$

$-2.500050$
.001413

1.394680

2. 101421

1.425367

.026677

$-.688485$

$-.540751$

1. 941093

$-.494837$

$-1.762698$

3. 449270

2.238179

3.562368

1.753253

4. 253428

4.155011

5.573277

6.530141

6.091274

5.297069

7.442622

6.809791

6.107582

4. 371599

5.206780

3. 361087

4.765336

5.088590

4.896122

6.264306

\section{endo-exo $S_{0}$ minimum}

$\mathrm{C}$
$\mathrm{C}$
$\mathrm{C}$
$\mathrm{C}$
$\mathrm{C}$
$\mathrm{C}$
$\mathrm{H}$
$\mathrm{H}$
$\mathrm{H}$
$\mathrm{H}$
$\mathrm{O}$
$\mathrm{C}$
$\mathrm{C}$
$\mathrm{H}$
$\mathrm{C}$
$\mathrm{H}$
$\mathrm{C}$
$\mathrm{C}$
$\mathrm{H}$
$\mathrm{H}$
$\mathrm{H}$

.180461

1.564574

2.147298

1.356157

$-.031998$

$-.623924$

$-.265659$

2.197491

$-.641460$

$-1.690919$

3. 494781

2. 042711

3. 355263

1.477010

4.150458

3.875940

5.529139

5.496080

6.095944

6.053051

6.506742
.033146

.140565

1.395417

2. 550402

2. 424795

1.174522

$-.936288$

$-.726800$

3.311441

1.090336

1.455897

3.838137

3. 874248

4.742573

2. 611635

4.810473

2.671127

2.863381

3.478570

1.749334

2.883068 
Supporting Information for Migani et al., The Ring Opening of Chromenes...

$\begin{array}{lrrr}\mathrm{H} & 1.764745 & 5.015615 & 3.796273 \\ \mathrm{H} & 1.970261 & 4.960679 & 2.055446 \\ \mathrm{C} & -2.195366 & 4.247404 & 2.284677 \\ \mathrm{C} & -3.074923 & 4.910406 & 3.346986 \\ \mathrm{H} & -2.557520 & 3.239090 & 2.107674 \\ \mathrm{H} & -2.291180 & 4.781593 & 1.344042 \\ \mathrm{H} & -4.112092 & 4.892924 & 3.028508 \\ \mathrm{H} & -3.020100 & 4.391401 & 4.298352 \\ \mathrm{H} & -2.806332 & 5.948015 & 3.513528\end{array}$

\section{endo-endo $S_{0}$ minimum}

C
C
C
C
C
C
H
H
H
H
O
C
C
H
C
H
C
C
H
H
H
H
H
C
C
H
H
H
H
H

exo-exo $S_{1}$ minimum $=M$

.113404
.091913
1.284362
2.504046
2.508218
1.322662
-.808733
-.829738
3.445770
1.340556
1.193349
3.717619
3.656203
4.653867
2.371156
4.542592
2.145466
2.077603
2.941268
1.218727
1.886833
3.005363
1.278647
2.360475
2.443013
1.451339
3.191116
2.440199
1.595793
3.348969

.113404
.091913
1.284362

.024477
-.110970

$-.054752$

.144275

.275758

.216106

$-.022393$

$-.257003$

.426352

.317513

$-.163274$

.203178

$-.016034$

.425941

$-.344919$

.018877

.602443

2.084978

.436270

.308711

2.678016

2.436925

2.275378

$-1.800697$

$-2.870454$

$-1.948192$

$-1.922984$

$-3.858173$

$-2.813237$

$-2.779536$
$-.016788$

1.370757

2. 091734

1.434381

.041187

$-.688380$

$-.567768$

1.903238

$-.465488$

$-1.758298$

3.433828

2.251030

3.571138

1.769944

4.291388

4.180911

5.478918

5.117168

6.201406

5.961737

6.006284

4. 678714

4.410761

4.786392

3.698348

5.361721

5.478193

4.148961

3.025544

3.109432
$-.012563$

$-.008505$

1. 236448

2.504626

2.469559

1.219465

$-.939891$

$-.916630$

3. 389125

1. 223461

1.166697

3. 677013
.052725

$-.051867$

$-.065393$

.043938

.149536

.149874

.053367

$-.120941$

.245678

.229703

$-.072087$

.098271
-. 021244

1. 399022

2. 098964

1. 424047

$-.013782$

$-.713616$

$-.560870$

1.965118

$-.559612$

$-1.784885$

3. 431999

2. 215121 
Supporting Information for Migani et al., The Ring Opening of Chromenes...

$\begin{array}{lrrr}\mathrm{C} & 3.623484 & -.075793 & 3.569403 \\ \mathrm{H} & 4.620990 & .291523 & 1.735097 \\ \mathrm{C} & 2.341306 & -.462353 & 4.256215 \\ \mathrm{H} & 4.519044 & -.042610 & 4.158372 \\ \mathrm{C} & 2.083891 & .315500 & 5.561382 \\ \mathrm{C} & 3.260611 & .502637 & 6.524071 \\ \mathrm{H} & 1.259198 & -.165234 & 6.079655 \\ \mathrm{H} & 1.729278 & 1.297079 & 5.268921 \\ \mathrm{H} & 2.908865 & .992453 & 7.426393 \\ \mathrm{H} & 3.724218 & -.429565 & 6.821597 \\ \mathrm{H} & 4.029039 & 1.135477 & 6.094835 \\ \mathrm{C} & 2.196270 & -1.992073 & 4.389115 \\ \mathrm{C} & 3.218548 & -2.720239 & 5.263343 \\ \mathrm{H} & 2.244053 & -2.400806 & 3.384686 \\ \mathrm{H} & 1.195525 & -2.192902 & 4.759558 \\ \mathrm{H} & 3.083468 & -3.792096 & 5.160415 \\ \mathrm{H} & 4.238941 & -2.492585 & 4.973323 \\ \mathrm{H} & 3.102884 & -2.482988 & 6.314320\end{array}$

\section{endo-exo $S_{1}$ minimum}

$\begin{array}{lrrr}\text { C } & 0.369252 & 0.170958 & -0.010057 \\ \text { C } & 0.173286 & 1.576261 & 0.113047 \\ \text { C } & -0.055130 & 2.143533 & 1.404004 \\ \text { C } & -0.078366 & 1.347753 & 2.605159 \\ \text { C } & 0.123106 & -0.072051 & 2.449648 \\ \text { C } & 0.341247 & -0.638968 & 1.152294 \\ \text { H } & 0.532731 & -0.269674 & -0.974558 \\ \text { H } & 0.191261 & 2.227846 & -0.738447 \\ \text { H } & 0.126506 & -0.703542 & 3.317944 \\ \text { H } & 0.490071 & -1.699341 & 1.066343 \\ \text { O } & -0.141372 & 3.475994 & 1.457354 \\ \mathrm{C} & -0.233784 & 2.014046 & 3.845684 \\ \mathrm{C} & -0.485990 & 3.354746 & 3.892589 \\ \mathrm{H} & -0.136851 & 1.449657 & 4.757162 \\ \mathrm{C} & -0.725360 & 4.150672 & 2.637951 \\ \mathrm{H} & -0.586638 & 3.868081 & 4.830931 \\ \mathrm{C} & -0.029931 & 5.515321 & 2.673269 \\ \mathrm{C} & 1.490083 & 5.453523 & 2.819413 \\ \mathrm{H} & -0.447521 & 6.087766 & 3.496107 \\ \mathrm{H} & -0.284110 & 6.046585 & 1.760695 \\ \mathrm{H} & 1.904354 & 6.456979 & 2.836471 \\ \mathrm{H} & 1.784045 & 4.958394 & 3.738840 \\ \mathrm{H} & 1.939682 & 4.918735 & 1.992069 \\ \mathrm{C} & -2.226392 & 4.274031 & 2.314231 \\ \mathrm{C} & -3.079685 & 4.988756 & 3.363687 \\ \mathrm{H} & -2.612028 & 3.269430 & 2.170593 \\ \mathrm{H} & -2.316997 & 4.783771 & 1.359581 \\ \mathrm{H} & -4.118121 & 4.996616 & 3.048601 \\ \mathrm{H} & -3.035646 & 4.489324 & 4.325699 \\ \mathrm{H} & -2.777906 & 6.021066 & 3.505160\end{array}$

\section{endo-endo $S_{1}$ minimum}

$\begin{array}{lrrr}\text { C } & .052647 & .164198 & -.014482 \\ \text { C } & .059197 & -.089844 & 1.387323 \\ \text { C } & 1.300011 & -.093007 & 2.095550\end{array}$


Supporting Information for Migani et al., The Ring Opening of Chromenes...

$\mathrm{C}$
$\mathrm{C}$
$\mathrm{C}$
$\mathrm{H}$
$\mathrm{H}$
$\mathrm{H}$
$\mathrm{H}$
$\mathrm{H}$
$\mathrm{O}$
$\mathrm{C}$
$\mathrm{C}$
$\mathrm{H}$
$\mathrm{C}$
$\mathrm{H}$
$\mathrm{C}$
$\mathrm{C}$
$\mathrm{H}$
$\mathrm{H}$
$\mathrm{H}$
$\mathrm{H}$
$\mathrm{H}$
$\mathrm{C}$
$\mathrm{C}$
$\mathrm{C}$
$\mathrm{H}$
$\mathrm{H}$
$\mathrm{H}$
$\mathrm{H}$
$\mathrm{H}$

\section{CP}

C

C

C

C

C

C

$\mathrm{H}$

H

$\mathrm{H}$

$\circ$

C

C

$\mathrm{H}$

C

$\mathrm{H}$

C

C

$\mathrm{H}$

$\mathrm{H}$

H

$\mathrm{H}$

C

C

$\mathrm{H}$

$\mathrm{H}$

$\mathrm{H}$
2.560338

2. 522720

1.278281

$-.871668$

$-.843838$

3.438861

1.281500

1.215992

3.736690

3.682321

4.684076

2.394965

4.574169

2. 127459

2. 019862

2. 924923

1.207614

1. 821254

2. 935278

1.209852

2.376433

2. 520316

1.444109

3.176877

2.492823

1.711743

3. 457253
.155828

.411386

.411214

.166736

$-.281080$

.602722

.602514

$-.335132$

.136963

-. 123710

.332075

$-.412313$

$-.139154$

.610219

2.061753

.518552

.323737

2.709814

2.402526

2.186338

$-1.834372$

$-2.959289$

$-1.959657$

$-1.902665$

$-3.922638$

$-2.938027$

$-2.893105$
1.447495

.028235

$-.680048$

$-.559180$

1.933114

$-.497877$

$-1.737120$

3. 406297

2.235342

3.572450

1.763104

4.293068

4.172990

5. 408522

4.944938

6.142060

5.909072

5.792964

4.474157

4.235870

4.876363

3.852893

5.418586

5.609185

4. 352644

3.131621

3. 311255
.000000

.000000
1.243589

2. 515338

2.483803

1.234363

$-.926858$

$-.910829$

3.406066

1. 240568

1.188060

3. 687644

3.631056

4.626154

2. 354496

4. 519449

2.100597

3.299337

1. 322523

1.683042

2. 950277

3.837156

4.005449

2. 200027

3.187424

2.277983

1.186736

3.025422

4.217841
.000000

.000000

.000000

.018078

$-.011298$

$-.011365$

$-.002585$

.002421

.009478

$-.014160$

.102834

.154378

.072666

.340044

$-.357487$

.169385

.309183

.489369

$-.253582$

1.288293

.865271

$-.431894$

1.213452

$-1.892493$

$-2.685126$

$-2.235552$

$-2.109725$

$-3.747514$

$-2.471416$
.000000

1.424118

2.127510

1. 446181

.005362

$-.695845$

$-.540496$

1.990241

$-.543854$

$-1.770077$

3. 458274

2. 228971

3.593091

1.734498

4.264482

4.185536

5.630537

6.569532

6.138395

5.425646

7.526075

6.759219

6.178947

4. 281588

5.136470

3.255556

4.606928

4.984365

4.871055

- S11 - 
Supporting Information for Migani et al., The Ring Opening of Chromenes...

\section{TS}

$\begin{array}{lr}\mathrm{C} & -2.0960700558 \\ \mathrm{C} & -2.0960700558 \\ \mathrm{C} & -0.8698344470 \\ \mathrm{C} & 0.4240209238 \\ \mathrm{C} & 0.3707896015 \\ \mathrm{C} & -0.8629234691 \\ \mathrm{H} & -3.0189546512 \\ \mathrm{H} & -3.0060287241 \\ \mathrm{H} & 1.2905054195 \\ \mathrm{H} & -0.8399975235 \\ \mathrm{O} & -0.9520725226 \\ \mathrm{C} & 1.5938213589 \\ \mathrm{C} & 1.5668625271 \\ \mathrm{H} & 2.5211156974 \\ \mathrm{C} & 0.3575318587 \\ \mathrm{H} & 2.3994425174 \\ \mathrm{C} & 0.0609052241 \\ \mathrm{C} & 1.2499815052 \\ \mathrm{H} & -0.7139077874 \\ \mathrm{H} & -0.3625564072 \\ \mathrm{H} & 0.8964902288 \\ \mathrm{H} & 1.7700955159 \\ \mathrm{H} & 1.9689169953 \\ \mathrm{C} & 0.0661779405 \\ \mathrm{C} & 1.0131463041 \\ \mathrm{H} & 0.1420443966 \\ \mathrm{H} & -0.9570554364 \\ \mathrm{H} & 0.7979704712 \\ \mathrm{H} & 2.0470211260 \\ \mathrm{H} & 0.9025779756 \\ & \end{array}$
0.3185922701
0.3185922701
0.3185922701
0.2557746580
0.2243333801
0.2619943741
0.3431644887
0.3473612326
0.2144443558
0.2499465444
0.4385582075
0.3698835111
0.3580394548
0.5533693304
$-0.0652883463$
0.7278243044
0.6172977998
0.8130482802
0.0543159530
1. 5891481818
1.2199547024
$-0.1114987058$
1. 5142925652
$-1.5725833840$
$-2.3985507783$
$-1.9204153723$
$-1.7368185587$
$-3.4542573109$
$-2.2328129702$
$-2.1712261990$

$$
\begin{array}{r}
-2.9273389323 \\
-1.5162129332 \\
-0.7960646123 \\
-1.4679466091 \\
-2.8861763673 \\
-3.5971820916 \\
-3.4719124325 \\
-0.9498224837 \\
-3.4398247132 \\
-4.6704761211 \\
0.4870656657 \\
-0.7007108565 \\
0.6826192015 \\
-1.2149229624 \\
1.3819465250 \\
1.2456489755 \\
2.7207602817 \\
3.6646199228 \\
3.2308067752 \\
2.4994868612 \\
4.6058338693 \\
3.8813493599 \\
3.2605995407 \\
1.3601607387 \\
2.2297077803 \\
0.3378050964 \\
1.6822365066 \\
2.1035375505 \\
1.9508316487 \\
3.2836424419
\end{array}
$$

$-.185865$

1.158197

1.991868

1.439599

.070248

$-.739975$

$-.804356$

1. 610659

$-.357344$

$-1.783859$

3. 214705

2. 244264

3. 611000

1. 716988

4.475784

4.075334

5.855167

6.687210

6.408417

5.742013 
Supporting Information for Migani et al., The Ring Opening of Chromenes...

$\begin{array}{lrrr}\mathrm{H} & 3.427824 & .772087 & 7.682267 \\ \mathrm{H} & 4.265073 & -.484707 & 6.790138 \\ \mathrm{H} & 4.337085 & 1.181970 & 6.248874 \\ \mathrm{C} & 2.111348 & -1.766130 & 4.240434 \\ \mathrm{C} & 2.967691 & -2.818937 & 4.963097 \\ \mathrm{H} & 2.102405 & -2.000798 & 3.186212 \\ \mathrm{H} & 1.087570 & -1.836059 & 4.592417 \\ \mathrm{H} & 2.577149 & -3.814480 & 4.782926 \\ \mathrm{H} & 3.992906 & -2.791906 & 4.609766 \\ \mathrm{H} & 2.978156 & -2.658907 & 6.03611\end{array}$

